\title{
Expression of RNA-Interference/Antisense Transgenes by the Cognate Promoters of Target Genes Is a Better Gene- Silencing Strategy to Study Gene Functions in Rice
}

\author{
Jing $\mathrm{Li}^{19}$, Dagang Jiang ${ }^{19}$, Hai Zhou ${ }^{1}$, Feng $\mathrm{Li}^{1}$, Jiawei Yang ${ }^{1}$, Laifa Hong ${ }^{1}$, Xiao Fu ${ }^{1}$, Zhibin $\mathrm{Li}^{1}$, Zhenlan \\ Liu ${ }^{1}$, Jianming $\mathrm{Li}^{2}$, Chuxiong Zhuang ${ }^{1 *}$
}

1 Key Laboratory of Plant Functional Genomics and Biotechnology, Education Department of Guangdong Province, South China Agricultural University, Guangzhou, People's Republic of China, 2 Department of Molecular, Cellular, and Developmental Biology, University of Michigan, Ann Arbor, Michigan, United States of America

\begin{abstract}
Antisense and RNA interference (RNAi)-mediated gene silencing systems are powerful reverse genetic methods for studying gene function. Most RNAi and antisense experiments used constitutive promoters to drive the expression of RNAi/antisense transgenes; however, several reports showed that constitutive promoters were not expressed in all cell types in cereal plants, suggesting that the constitutive promoter systems are not effective for silencing gene expression in certain tissues/ organs. To develop an alternative method that complements the constitutive promoter systems, we constructed RNAi and/ or antisense transgenes for four rice genes using a constitutive promoter or a cognate promoter of a selected rice target gene and generated many independent transgenic lines. Genetic, molecular, and phenotypic analyses of these RNAi/ antisense transgenic rice plants, in comparison to previously-reported transgenic lines that silenced similar genes, revealed that expression of the cognate promoter-driven RNAi/antisense transgenes resulted in novel growth/developmental defects that were not observed in transgenic lines expressing constitutive promoter-driven gene-silencing transgenes of the same target genes. Our results strongly suggested that expression of RNAi/antisense transgenes by cognate promoters of target genes is a better gene-silencing approach to discovery gene function in rice.
\end{abstract}

Citation: Li J, Jiang D, Zhou H, Li F, Yang J, et al. (2011) Expression of RNA-Interference/Antisense Transgenes by the Cognate Promoters of Target Genes Is a Better Gene-Silencing Strategy to Study Gene Functions in Rice. PLoS ONE 6(3): e17444. doi:10.1371/journal.pone.0017444

Editor: Roland Roberts, King's College London, United Kingdom

Received August 30, 2010; Accepted February 4, 2011; Published March 3, 2011

Copyright: (C) $2011 \mathrm{Li}$ et al. This is an open-access article distributed under the terms of the Creative Commons Attribution License, which permits unrestricted use, distribution, and reproduction in any medium, provided the original author and source are credited.

Funding: This work was supported by the National Basic Research Program of China (2005CB120802, http://www.973.gov.cn/English/Index.aspx), the National High Technology Research and Development Program of China (2007AA10A144, http://www.most.gov.cn/eng/programmes1/200610/t20061009_36225.htm), the National Natural Science Foundation of China (30470983, http://www.973.gov.cn/English/Index.aspx) and Genetically Modified Organisms breeding Major Projects (2008ZX08001-004, http://www.most.gov.cn/index.htm). The funders had no role in study design, data collection and analysis, decision to publish, or preparation of the manuscript.

Competing Interests: The authors have declared that no competing interests exist.

*E-mail: zhuangcx@scau.edu.cn

9 These authors contributed equally to this work.

\section{Introduction}

Plant genomic research has made remarkable progress in recent years. The genome sequence of a plant provides the foundation for detailed functional characterization of plant genes [1]. Rice was the first crop plant to have its complete genome sequenced [2]. Although 56,797 genes have been annotated from sequencing of the rice genome [3,4], the functions of $>60 \%$ of these predicted genes are unknown. Therefore, one of the most challenging goals of the rice functional genomics is to characterize the functions of these unknown rice genes.

Reverse genetics is a powerful tool for assessing gene function [5], and several reverse genetics approaches have been developed in recent years for functional genomic studies. Transfer DNA (TDNA) insertional mutagenesis that creates loss of function mutations [6] is a very effective reverse genetics approach in studying gene functions. Although T-DNA insertional mutagen has been widely used, it has several disadvantages. One common drawback is complex organizations of many T-DNA inserts, resulting in an overall $40 \%$ to $50 \%$ failure rate in identifying the exact T-DNA insertional site [7]. Besides, T-DNA exhibits certain integration preference and may therefore not saturate the entire rice genome [8]. As a result, only 27,551 rice genes were found to be mutated by T-DNA insertions from collections of $>400,000$ independent rice T-DNA lines [8]. In addition, T-DNA insertion may lead to lethal phenotypes, preventing genetic studies of gene functions, or cause no observable phenotype due to functional redundancy of homologous genes.

Several alternative reverse genetic approaches to study gene function, such as RNA interference (RNAi) and antisense RNA technology could circumvent the limitations of T-DNA insertional mutagenesis. In RNAi technology, the introduction of doublestranded RNAs (dsRNAs) into cells inhibits the expression of the corresponding endogenous gene at transcriptional and posttranscriptional levels [9]. RNAi could silence the expression of an endogenous target gene without altering its gene structure or producing the permanent loss of gene function. The partial gene silencing-effect of the RNAi and antisense strategies could avoid potential lethality of a T-DNA insertional mutation. In addition, RNAi/antisense-initiated gene silencing could simultaneously inhibit the expression of several homologous genes, thus overcoming potential gene redundancy problems. These advantages have made 
the RNAi and antisense RNA strategies the method of choice for studying gene functions in plants in recent years.

The choice of promoter is a very important factor in RNAi and antisense RNA strategies. The most commonly used promoters in RNAi and antisense strategies are constitutive promoters, such as the $35 S$ promoter from cauliflower mosaic virus ( $p$ CaMV35S) [10] and the promoter from the maize Ubiquitin-1 gene ( $p$ Ubil) [11]. Without species restriction, constitutive promoters drive high expression in virtually all tissues/organs of transgenic plants independently of tissue/organ-specific regulators or developmental/environmental signals. However, the constitutive promoterdriven expression of an RNAi/antisense-transgene could cause pleiotropic phenotypes or embryo lethality by silencing the expression of the target gene and its homologs, thus making it extremely difficult to study the functions of the target gene or to define a causal relationship between a silenced gene and the observed phenotypic alterations. On the other hand, recent studies revealed that constitutive promoters are not active in all cell types, especially in cereal crops $[12,13]$. Therefore, gene functions cannot be fully defined, as the expression pattern of an RNAi/ antisense transgene might not completely overlap with that of its target gene.

Regulated promoters such as organ/tissue- or developmental stage-specific promoters $[14,15]$ and physically/chemically-inducible promoters $[16,17,18,19,20]$ have been used in the past to better control the expression of an RNAi/antisense transgene avoiding the adverse effects of constitutive promoters. However, these promoters have their own limitations as an RNAi/antisensetransgene driven by a regulated promoter will only be expressed in certain tissues/organs, at specific developmental stages, or in response to a unique chemical/physical signal but has no effect on the target gene in other relevant tissues/organs at certain important developmental stages [21].

By contrast, a cognate promoter of a target gene should drive the expression of a gene-knockdown RNAi/antisense-transgene in the native expression domains of the targeted endogenous gene, which could overcome many of the known limitations of constitutive/regulated promoters in driving the expression of gene-silencing transgenes to define the biological functions of their corresponding endogenous genes.

In this study, we investigated the effectiveness of constitutive/ cognate promoter-driven RNAi/antisense-transgene in causing growth/developmental phenotype in transgenic rice plants. Four rice genes, Pyruvate Dehydrogenase Kinase 1 and 2 (OsPDK1 and OsPDK2), Silencing Information Regulator 2 (OsSRT1), and Metallothio$n e i n 2 b(O s M T 2 b)$, were selected for our studies. The physiological functions of these four genes were previously studied by gene silencing using constitutive promoter-driven RNAi/antisense transgenes [22,23,24], however, our studies using the cognate promoter-driven $\mathrm{RNAi}$ /antisense transgenes revealed additional functions of these genes in regulating rice growth/development. Our investigation with the two $O S P D K$ genes also showed that the cognate promoter approach could specifically reduce the transcript level of one member gene without affecting the expression of other members of a gene family.

\section{Results}

The cognate promoter-driven expression of an RNAitransgene revealed novel physiological functions of OsMT2b

Metallothioneins (MTs) are a family of low-molecular weight, cysteine rich intracellular proteins that are thought to play important roles in metal tolerance, detoxification, and homeostasis in plants via binding heavy metals $[22,25,26]$. The rice genome encodes 15 MT proteins that could be classified into four types [22]. OsMT2b, a type-2 MT, scavenges reactive oxygen species $[22,27]$. Earlier studies using transgenic rice plants in which $O s M T 2 b$ was silenced by an OsMT2b-RNAi transgene driven by the maize Ubi promoter showed that OsMT2b participates in epidermal cell death [28] and is involved in root development and seed embryo germination by modulating the endogenous cytokinin level [22].

To better understand the physiological functions of OsMT2b, we generated an $O s M T 2 b$ RNAi transgene driven by the cognate promoter of the endogenous OsMT2b gene (Figure 1A) and transformed the resulting $p O s M T 2 b:: O s M T 2 b$-RNAi transgene into wild-type rice plants. Ten independent transgenic lines were obtained and carefully analyzed, among which 6 transgenic lines exhibited phenotypic variations in the $\mathrm{T}_{0}$ generation. RNA blot analyses found that the expression of the endogenous OsMT2b gene was significantly reduced in two independent $p O s M T 2 b::$ OsMT2b-RNAi transgenic lines exhibiting the growth defects (Figure 2A), while segregation analysis of $\mathrm{T}_{1}$ progeny of several $\mathrm{T}_{0}$ lines carrying single-copy transgene revealed a 3:1 ratio for normal individuals vs. abnormal individuals. Analyses of the morphological/developmental defects of the 6 independent $T_{0}$ transgenic lines and their offspring not only confirmed previously reported phenotypic alterations, including smaller mature embryos and reduced thickness of scutellum of embryos (Figure 2B), but also discovered novel growth phenotypes such as smaller spikelets, lower percentage of seed setting, and smaller seeds at the bottom of spikes (Figure 2C). Our study thus revealed a functional role of OsMT2b in spikelet/seed development, suggesting that the cognate promoter-driven gene silencing is a better strategy than the constitutive promoter-driven gene silencing to study gene functions in rice.

\section{Silencing of the rice OsSRT1 gene by cognate promoter- driven OsSRT1-RNAi or OsSRT1-antisense transgenes}

To further confirm our discovery, we generated a cognate promoter-driven RNAi transgene for another rice gene, which encodes a protein homologous to the SILENT INFORMATION REGULATOR2 (SIR2), a highly conserved $\mathrm{NAD}^{+}$-dependent protein deacetylase $[29,30]$. The rice genome encodes two SIR2related proteins, named OsSRT1 and OsSRT2 [23,31]. An earlier study showed that transgenic rice plants in which OsSIRT1 was silenced by an OSSRT1-RNAi transgene driven by the CaMV35S promoter exhibited brown dots on leaves, which became larger at later stages, leading to premature leaf senescence [23].

Despite numerous attempts, we were unable to generate a single pOsSRT1::OsSRT1-RNAi (Figure 1B) transgenic rice line from the OsSRT1-RNAi transgene-transformed calli. We suspected that the RNAi-mediated silencing of OSSRT1 in its native expression domains prevented transformed calli to regenerate. To test our hypothesis, we performed a Southern blot analysis with genomic DNAs isolated from antibiotic-resistant calli and found that these hygromycin-resistant calli carried the hygromycin-B-phosphotransferase gene, the antibiotic marker gene of the pOsSRT1:: OSSRT1-RNAi plasmid and originated from different transformation events (data not shown). We also performed RNA blot analysis using total RNAs isolated from hygromycin-resistant and control calli and found that the OSSRT1 transcript level was significantly reduced in hygromycin-resistant calli (Figure 3A). Given the successful generation of multiple transgenic lines when an OsSRT1-RNAi transgene was driven by the CaMV35S promoter [23], our use of a cognate promoter-driven RNAitransgene revealed a novel role of OSSRT1 in tissue regeneration. 


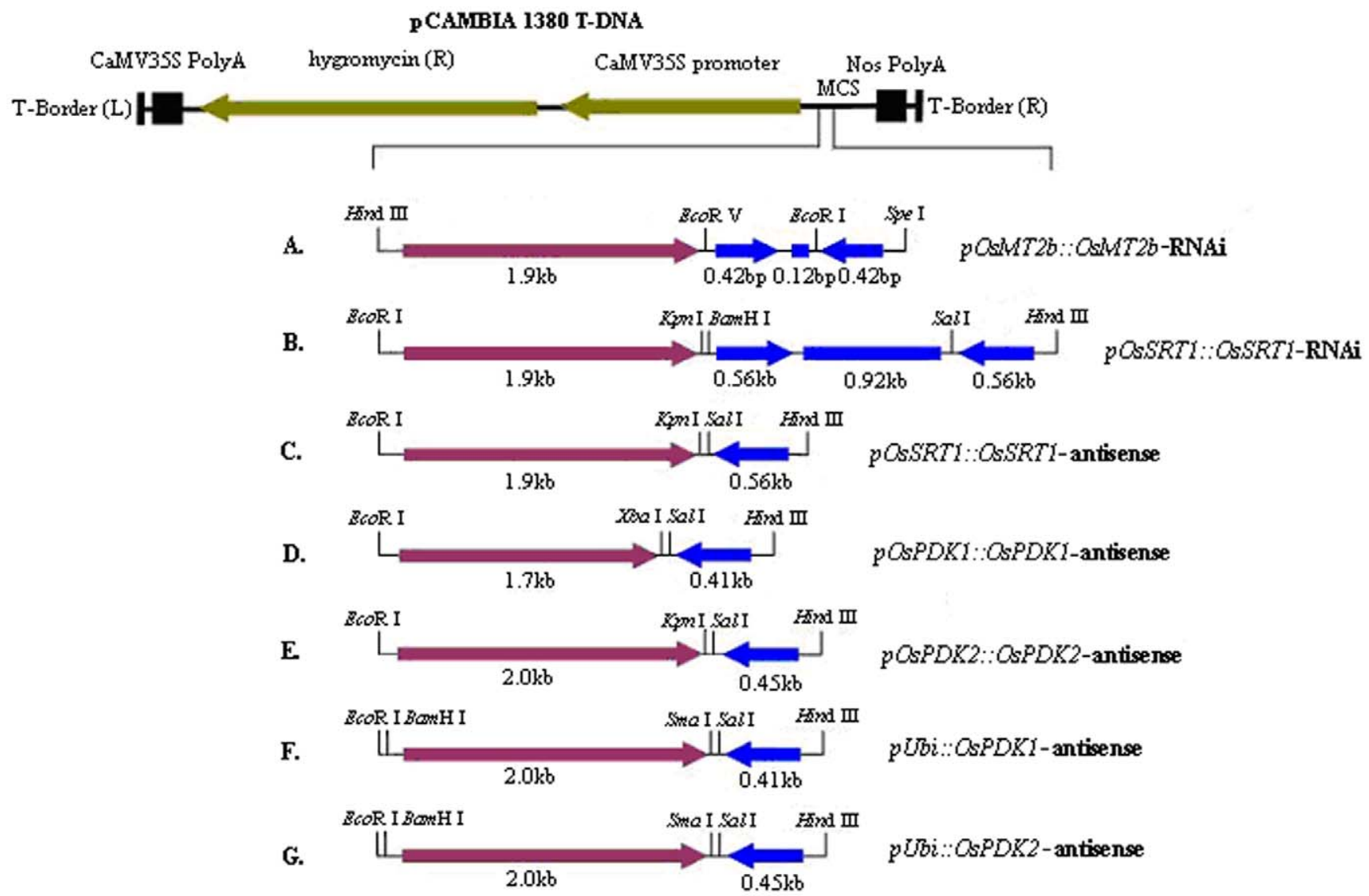

Figure 1. Schematic presentation of the constructed RNAi/antisense transgenes. (A, B) Positions and orientations of independently amplified genomic/cDNA fragments for generating pOsMT2b::OsMT2b and pOsSRT1::OsSRT1 RNAi transgenes. (C-E) Schematic presentation of antisense transgenes of OsSRT1 (C), OsPDK1 (D) and OsPDK2 (E) driven by their cognate promoters. (F, G) Schematic presentation of the pUbi::OsPDK1 (F) and pUbi::OsPDK2 (G) antisense transgenes. Purple arrows represent promoters, blue right arrows indicate sense fragments, blue left arrows mean antisense fragments, and blue bars denote introns.

doi:10.1371/journal.pone.0017444.g001

Because no transgenic plants were obtained with the pOsSRT1:: OSSTR1-RNAi transgene, we created a pOsSRT1::OSSRT1 antisense transgene carrying the cognate promoter of the endogenous OSSRT1 gene (Figure 1C), as an antisense transgene is less effective in triggering gene silencing. A total of 12 independent transgenic lines were produced but none of them exhibited any observable growth alteration. However, at least $5 \mathrm{~T}_{0}$ lines segregated out $\mathrm{T}_{1}$ individuals displaying developmental defects with a $3: 1$ ratio of normal plants vs. defective individuals (data not shown). Further genetic studies suggested that the defective $T_{1}$ plants are likely homozygous for the $p O S S R T 1:: O s S R T 1$-antisense transgene as they failed to segregate out normal plants in 4 subsequent generations. Two homozygous pOsSRT1::OSSRT1-antisense lines were selected to determine the gene silencing effect of the cognate-promoterdriven antisense transgene.

Although RT-PCR analysis detected no significant changes in the OsSRT1 transcript level (Figure 3B-a), our immunoblot experiment showed that the OsSRT1 protein abundance in the two pOSSRT1::OsSIRT1-antisense transgenic lines was significantly reduced (Figure 3B-d). Consistent with the known function of the yeast/mammalian SIR2 proteins in deacetylating the acetylated lysine-9 residue on histone 3 (H3K9) [23], an immunoblot assay using antibodies raised against the acetylated $\mathrm{H} 3 \mathrm{~K} 9$ revealed the increased H3K9 acetylation in the two selected transgenic lines (Figure 3B-e) , further supporting a reduction of OsSRT1 abundance in the two selected transgenic lines. These homozygous
pOSSRT1::OsSRT1-antisense transgenic rice plants not only displayed brown spots on the leaves and early senescence symptom (Figure 3C), which are similar to what were previously observed on pCaMV35S::OsSRT1-RNAi transgenic plants [23], but also exhibited additional growth/developmental abnormalities, such as decreased tillering capacity and lower seed setting (Figure 3C and data not shown). Our studies using pOsSRT1::OsSRT1-RNAi/ antisense transgenes therefore further supported our conclusion that expression of RNAi/antisense transgene using a cognate promoter of the target gene is a better silencing strategy in revealing its physiological functions in rice.

Direct comparison of the phenotypic differences of constitutive and cognate promoters in driving the expression of antisense transgenes in rice

To directly compare the differential effects of constitutive and cognate promoters on silencing rice genes, we created two antisense transgenes each for two highly-homologous rice genes encoding pyruvate dehydrogenase kinase 1 and 2 (OsPDK 1 and OsPDK2), one using the maize Ubi promoter and the other with the cognate promoters of the $O s P D K$ genes (Figure 1D-1G). An earlier study showed that silencing the OsPDK1 gene by a CaMV35S promoter-driven OsPDK1-RNAi transgene resulted in a weak dwarf phenotype in transgenic rice plants [24].

Transformation of $p O s P D K 1:: O s P D K 1$ and $p O s P D K 2:: O s P D K 2$ antisense transgenes resulted in generation of 16 and 13 

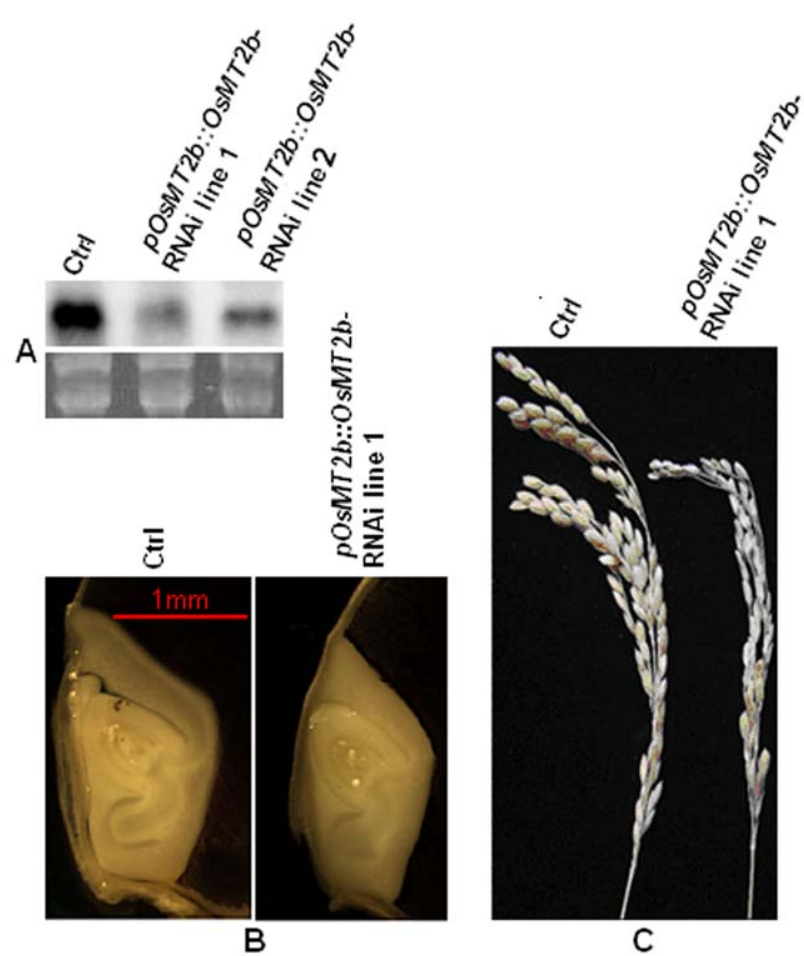

Figure 2. Phenotypic and RNA blot analyses of primary OsMT2b-RNAi transgenic lines. A. RNA blot analysis of the endogenous OsMT2b transcript. Twenty $\mu \mathrm{g}$ of total RNAs isolated from two independent pOsMT2b::OSMT2b-RNAi transgenic lines ( 1 and 2 ) and the control line (Ctrl) were separated on denaturing agarose gel, stained with ethidium bromide, transferred to a nylon membrane, hybridized with $\alpha{ }^{32}$ P-labeled OsMT2b cDNA fragment, and analyzed by autoradiography (upper panel). The lower panel shows the ethidium bromide-stained ribosomal RNAs used as a loading control. B. Comparison of the seed embryo between a representative OsMT2bRNAi transgenic line (1) and the control (Ctrl). Scale Bar $=1 \mathrm{~mm}$. C. Phenotypic comparison of panicles/spikelets between the representative pOsMT2b::OsMT2b-RNAi transgenic line (1) and the control (Ctrl). doi:10.1371/journal.pone.0017444.g002

independent transgenic lines of Zhonghua 11, respectively. Both transgenes caused two types of growth alterations. The first one is slightly-reduced plant height $(\sim 10 \%$ reduction compared to the control), resembling that of the previously-reported $p U b i:$ : OsPDK1-RNAi transgenic lines [24]. The other type of growth defects included severe dwarfism $(\sim 90 \%$ shorter than the control), single tillering, and semi-sterility (Figure $4 \mathrm{~A}$ and $4 \mathrm{~B}$ ), which were not observed in $p 35 S:: O s P D K 1$-RNAi transgenic rice plants. RT-PCR analysis revealed a slight reduction of the $O s P D K$ transcript abundance in weakly dwarfed transgenic plants but detected no $O S P D K$ transcripts in severely dwarfed lines (Figure 4G and 4D). Interestingly, despite high sequence similarity between the two $O S P D K$ genes, the antisense-triggered gene silencing was quite specific as the transcript levels of $O s P D K 1$ and $O S P D K 2$ were not obviously changed in OsPDK2-antisense and $O s P D K 1$-antisense transgenic plants, respectively (Figure $4 \mathrm{E})$. Consistently, the severely-dwarfed $p O s P D K 1:: O s P D K 1$ and pOsPDK2::OsPDK2-antisense transgenic lines also exhibited unique phenotypes. The pOsPDK1::OsPDK1-antisense lines had longer life cycle than the control plants with pale yellow leaves, whereas $p O s P D K 2:: O S P D K 2$-antisense dwarfs had shorter life cycle than the control with darker green leaves (Figure $4 \mathrm{~A}$ and $4 \mathrm{~B}$ ), revealing different physiological functions for two highly homologous rice proteins.

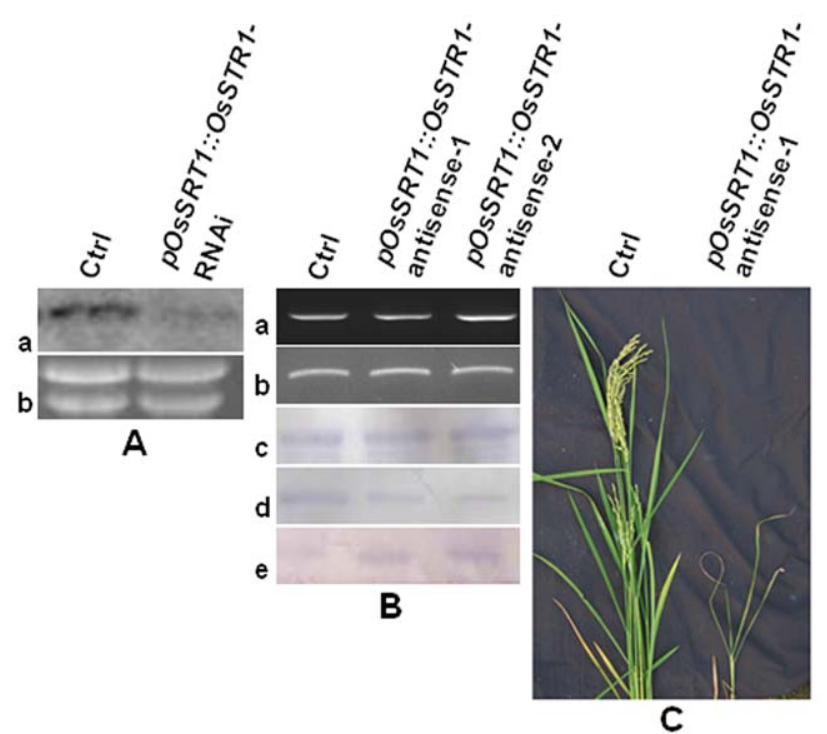

Figure 3. Phenotypic, molecular, and biochemical analyses of primary OSSRT1 antisense transgenic lines. A. RNA blot analysis of the OsSRT1 transcript. Twenty $\mu \mathrm{g}$ of total RNAs isolated from calli derived from the control rice plant (Ctrl) and hygromycin-resistant calli transformed with the POSSRT1::OSSRT1-RNAi transgene were separated on denaturing agarose gel, stained with ethidium bromide, transferred to a nylon membrane, hybridized with $\alpha^{-32}$-labeled OSSRT1 CDNA fragment, and analyzed by autoradiography(a). The lower panel shows the ethidium bromide-stained ribosomal RNAs used as a loading control (b). B. The expression of the pOsSRT1::OsSRT1-antisense transgene had no effect on the OsSRT1 mRNA level but significantly reduced the OsSRT1 protein abundance. POSSRT1::OsSRT1-antisense-1 and -2 are two independent OsSRT1-antisense transgenic lines. a) RTPCR analysis of the transcript abundance of the endogenous OsSRT1 gene (see Materials and Methods for experimental details). b) $\beta$-actin was used as a loading control. c-e) Immunoblot analysis of the protein abundance of Tubulin (c), OsSRT1(d), and the level of H3K9 acetylation(e). Equal amounts of protein crude extracts were separated by SDS-polyacrylamide gel electrophoresis, transferred to nitrocellulose filters, and analyzed by immunoblotting with antibodies against Tubulin (for loading control), OsSRT1, and acetylated Lys-9 residue of histone 3 (H3K9). C. Phenotypic comparison between a representative pOsSRT1::OsSRT1 antisense transgenic line (1) and a wild-type control (Ctrl).

doi:10.1371/journal.pone.0017444.g003

By contrast, expression of either $O s P D K$-antisense transgene driven by the constitutively-active $U b i$ promoter failed to cause extreme dwarfism but only resulted in the semidwarf phenotype ( $\sim 30 \%$ shorter than control plants) (Figure 5$)$, which is slightly stronger than that caused by the expression of pUbi::OsPDK1RNAi transgene [24]. Consistently, RT-PCR analysis revealed a slight reduction of $O S P D K 1$ or OSPDK2 transcript level in the pUbi::OsPDK-antisense transgenic lines (Figure 4C and 4D). As expected from the failure of the $p U b i: \because O s P D K$-antisense transgenes to cause strong dwarfism, no obvious phenotypic difference was observed between pUbi:OsPDK1- and pUbi:OsPDK2-antisense transgenic plants. Taken together, our direct comparison study clearly demonstrated the superiority of the cognate promoterdriven transgenes in silencing the corresponding endogenous rice genes to reveal their physiological functions.

\section{Discussion}

In this study, we investigated the differential effects of constitutive promoter-driven and cognate promoter-driven RNAi/antisense transgenes on gene silencing and causing growth/developmental 


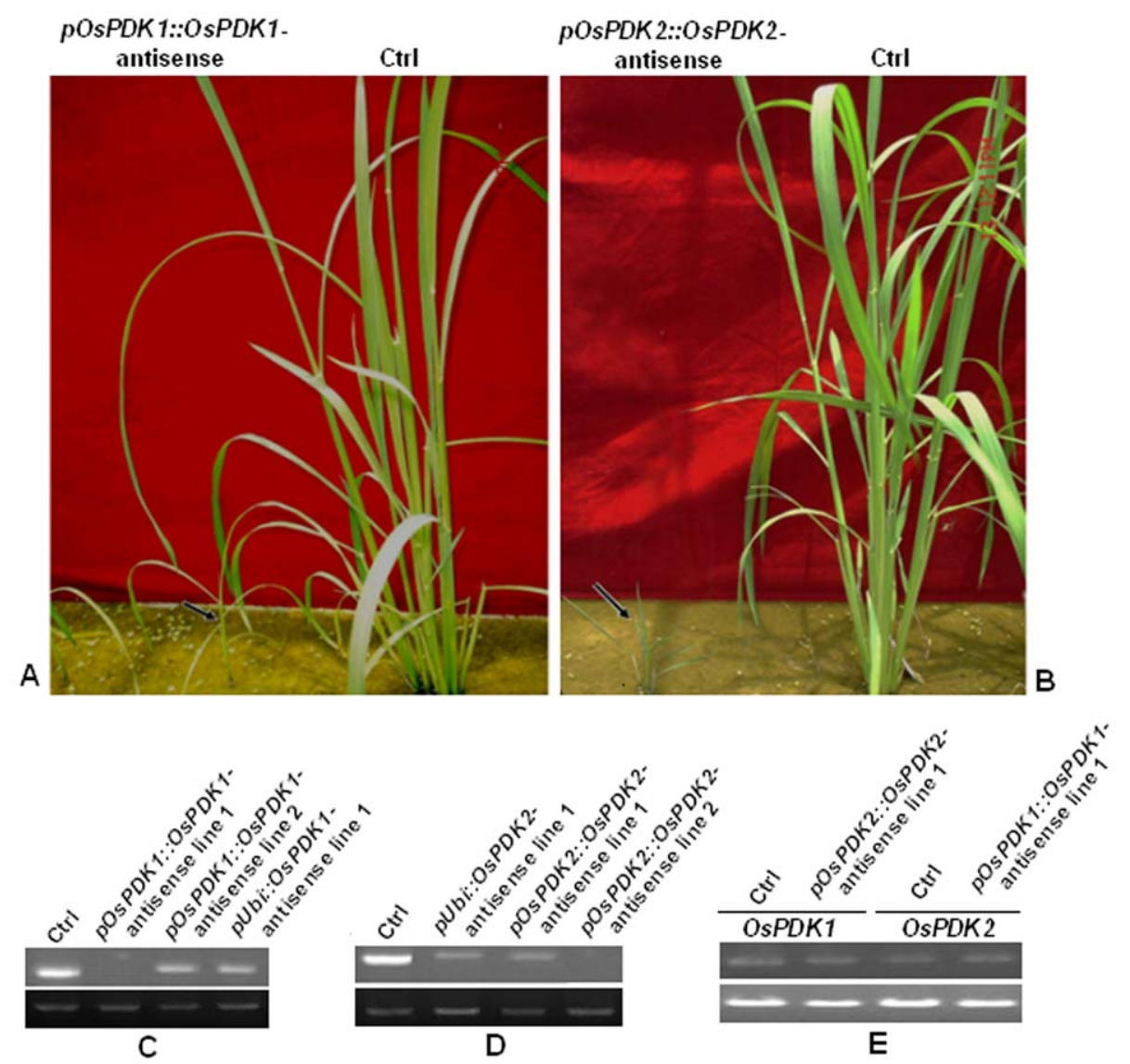

Figure 4. Similar and different growth/developmental defects in $p O s P D K:: O s P D K$-antisense transgenic plants. A. Phenotypic comparison between a representative pOsPDK1::OsPDK1-antisense transgenic line (indicated by black arrow) and the wild-type control (Ctrl) of the same developmental age (booting stage). B. Phenotypic comparison between a representative POSPDK2::OsPDK2-antisense transgenic line (indicated by black arrow) and the wild-type control (Ctrl) of the same developmental age (booting stage). C-E. RT-PCR analysis of the transcript abundance of the endogenous OsPDK1 and OsPDK2 genes in various pUbi/pOsPDK::OSPDK-antisense transgenic plants. Equal amounts of total RNAs isolated from the wild-type control (Ctrl) and selected transgenic plants were converted into $1^{\text {st }} \mathrm{CDNAs}$. Half microliter of the resulting $1 \mathrm{st}$-strand $\mathrm{cDNAs}$ was used as templates for PCR-amplification using gene-specific primers (see Materials and Methods for details) of the transcripts of the endogenous OsPDK1 (C plus lanes 1 and 2 in E) and OsPDK2 (D plus lanes 3 and 4 in E) genes. RT-PCR analysis of the rice $\beta$-actin gene (the lower strip in each panel) was used as a control.

doi:10.1371/journal.pone.0017444.g004

defects in transgenic rice plants. By comparing the growth/ developmental phenotypes of our transgenic plants with those of previously reported transgenic lines, we found that the expression of the cognate promoter-driven RNAi/antisense transgenes often gave rise to growth/developmental defects that were not observed on transgenic lines expressing constitutive promoter-driven RNAi/ antisense transgenes of the same target genes. For example, some pOsPDK1::OsPDK1-antisense transgenic lines were severe dwarfs with yellow leaves, which were not observed in previously reported pUbi/p35S::OsPDK1- antisense transgenic rice plants [24]. On the other hand, the use of a cognate promoter-driven RNAi/antisense transgene could avoid potential lethal phenotype caused by expression of a constitutive promoter-driven RNAi/antisense transgene. For example, an earlier study reported that strong silencing of the OSSRT1 gene caused a postembryonic lethal phenotype in $p 35 S:: O s S R T 1-R N A$ i transgenic plants [23], whereas no such a phenotype was observed in our pOSSRT1::OsSRT1antisense transgenic lines. Our results demonstrated that gene silencing using cognate promoter-driven $\mathrm{RNAi}$ /antisense transgenes was a more effective and physiologically relevant approach than that driven by constitutive promoters to define the gene functions in rice. We have so far adopted this approach to investigate the physiological functions of more than 20 rice genes (unpublished data).

Antisense RNA, with the formation of antisense/target dsRNA, is a gene silencing mechanism resulting in mRNA degradation or affecting mRNA translation [32,33]. It has been reported that the binding position of the antisense RNA may determine genesilencing mechanisms $[34,35]$. Antisense RNAs binding to the $3^{\prime}$ untranslated region ( $3^{\prime}$-UTR) represses translation [32], whereas antisense RNAs pairing with the 5' UTR of the target gene could cause mRNA degradation. The full-length of OSSRT1 (NM 001058878 ) cDNA is $1891 \mathrm{bp}$, and the predicted antisense transcript of the OSSRT1-antisense transgene would hybridize to the region near the 3 '-end, between nucleotides 1206 and 1770, of the endogenous OsSRT1 transcript. In pOsSRT1::OsSRT1-antisense transgenic plants, the transcript level of the endogenous OsSRT1 gene was not obviously changed but the OsSRT1 protein level and its predicted histone deacetylase activity were significantly reduced. The same antisense-transgene construction strategy was used for 8 additional rice genes, and similar effects on the abundance of the endogenous target genes and their protein products were observed (data not shown). The full-length of OSPDK1 (NM_001056731.1) and OsPDK2 (NM_001066936.1) cDNAs are 1535 bp and1480 bp, 


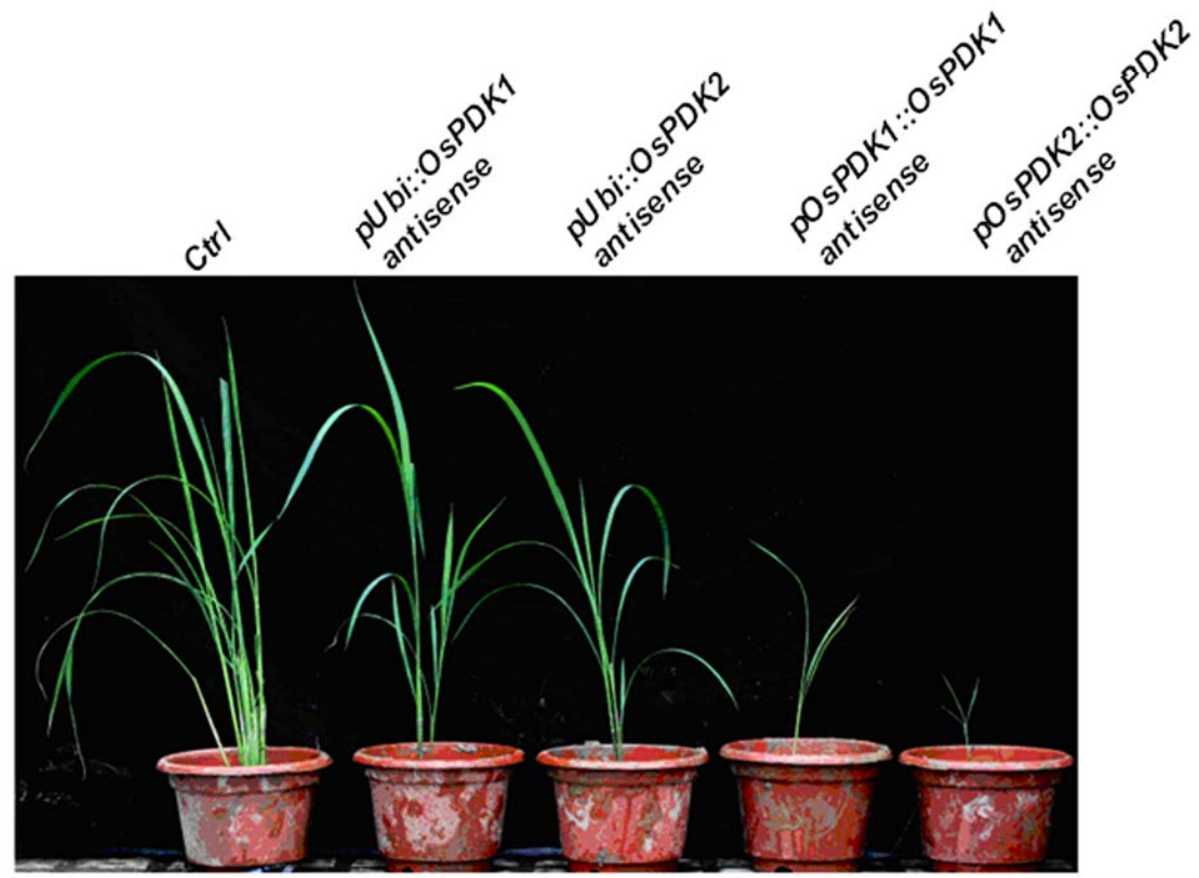

Figure 5. Phenotypic comparison between pOsPDK::OsPDK-antisense and pUbi::OsPDK-antisense transgenic plants. Shown here from left to right are six-week old soil-grown rice plants of the wild-type control (Ctrl) and a representative transgenic line carrying an antisense transgene of pUbi::OsPDK1, pUbi::OsPDK2, pOsPDK1::OsPDK1 and pOsPDK2::OsPDK2. doi:10.1371/journal.pone.0017444.g005

respectively. The cDNA fragments used for making antisense transgenes of $O S P D K 1$ and $O S P D K 2$ were derived from the regions spanning 434-845 bp and 153-594 bp near the 5' ends of OsPDK1 and $O s P D K 2$ cDNAs, respectively. In $p O s P D K 1:: O s P D K 1$ and pOsPDK2::OsPDK2-antisense transgenic progeny, the mRNA levels of the corresponding target genes decreased significantly. Our studies thus further supported an earlier hypothesis that antisense RNA directed against the 5' UTR often results in degradation of the target mRNA whereas antisense RNA targeted near the 3' UTR causes translational inhibition.

Consistent with earlier discoveries that the gene-silencing efficiency of antisense transgene is lower than that of RNAitransgene, growth/developmental defects were only observed in the homozygous progeny of OsSRT1/OsPDK1/OsPDK2-antisense transgenic lines. However, such a lower gene-silencing efficiency could be useful to avoid lethal phenotypes of RNAi-induced strong gene silencing. For example, despite numerous attempts and successful generation of antibiotic-resistant calli with an pOSSRT1::OsSRT1RNAi transgene, no single pOsSRT1::OsSRT1-RNAi transgenic plants was regenerated; however, we were quite successful in generating pOsSRT1::OsSRT1-antisense transgenic lines with reduced transcript level of the endogenous OSSRT1 gene. We suggest that the antisense-mediated gene-silencing technology might be more appropriate than the RNAi technology to study rice genes that play roles in early stage of plant growth/development.

Gene redundancy is a major obstacle in functional genomic studies. $53 \%$ and $68 \%$ of the non-transposable element-related genes in rice and Arabidopsis are grouped into paralogous gene families, respectively [36]. Although family members show high sequence homology at the nucleic acid level, they often have different expression patterns and biological functions. Genesilencing using a constitutive promoter-drive RNAi/antisense transgene could simultaneously knockdown the intended target gene and its potential homologs [37], making is extremely difficult in assigning a given biological function to a member of that gene family. For example, a recent report showed that four members of OsAGO1 gene family, OsAGO1a, OsAGO1b, OsAGO1c, and OsAGO1d, are highly similar in sequence with each other [38], and their transcript levels were all significantly reduced by the expression of a constitutive promoter-driven OsAGO1-RNAi transgene. In this work, we studied two members of the $O s P D K$ gene family, $O s P D K 1$ and $O s P D K 2$, which share $82 \%$ similarity at the nucleotide level. OsPDK1 is expressed in mature leaves, while OsPDK2 is mainly expressed in actively-growing tissues. As expected, no phenotypic difference was observed in pUbi:: OsPDK1/pUbi::OsPDK2-antisense transgenic lines, making it difficult to define the physiological function for each $O S P D K$ gene. However, transgenic plants in which the OsPDK1/OsPDK2antisense transgene was driven by the corresponding cognate promoter displayed different phenotypes. The pOsPDK1::OsPDK1antisense transgene caused yellowish leaf color and longer life cycle, whereas the expression of the $p O s P D K 2:: O s P D K 2$-antisense transgene resulted in darker green leaf color and a shortened life cycle with precocious flowering. Our results thus suggested that the expression of an antisense transgene by the cognate promoter of its target gene might be a better strategy to study the physiological functions of gene families.

\section{Materials and Methods}

\section{Plant and Other Experimental Materials}

Rice (Oryza sativa L. ssp. Faponica) cv. Zhonghua 11 was used for all experiments. Transgenic plants were grown in a greenhouse with normal daylight illumination. Escherichia coli DH10B and Agrobacterium tumefaciens strain EHA105 were used for cloning and transformation experiments. pCAMBIA1380 was used as the binary vector for Agrobacterium-mediated transformation [39]. 


\section{Plasmid Construction}

Two RNAi transgenes (OsSRT1 and OsMT2b) and three antisense transgenes (OsSRT1, OsPDK1 and OsPDK2) were constructed (Text S1). These 5 transgenes were driven by the cognate promoters of the corresponding target genes. To directly investigate the differential effect of cognate promoters and constitutive promoters on gene silencing, OsPDK1 and OsPDK2 antisense transgenes driven by the maize $p U b i$ promoter were also constructed. Primers were designed based on published cDNA sequences of OsSRT1, OsMT2b, OsPDK1 and OsPDK2 (Table 1) and were used to amplify gene-specific cDNA fragments from total RNAs isolated from Zhonghua 11. The published genome sequences were also used to locate the $2.0-\mathrm{kb}$ genomic fragment immediately upstream of the annotated ATG start codon for each gene (Table 2), which were amplified by PGR using the primer pairs listed in Table 1 and used as cognate promoters for RNAi/ antisense transgene construction. The intron fragments of RNAi transgenes were directly amplified the genomic DNA of Zhonghua 11 (Figure 1A and 1B). Each of the constructed transgenes was fully sequenced to ensure no PCR error before being transformed into Agrobacterial cells.

\section{Plant transformation}

To investigate the effectiveness of generated RNAi/antisense transgenes in silencing their target genes, these transgenes was then transformed into the A. tumefaciens strain EHA105, which were used to transform rice calli generated from mature dry seeds of Zhonghuall following a previously described protocol [39]. Tranformed calli were allowed to generate $\mathrm{T}_{0}$ plants. After further analyses, they were transferred into soil to produce $\mathrm{T}_{1}$ seeds for the generation of $\mathrm{T}_{1}$ transgenic lines.

\section{RNA preparation}

Total RNAs were extracted using the Trizol method (Invitrogen) according to the manufacturer's protocols. Briefly, $0.1 \mathrm{~g}$ plant tissues from leaves and spikelets of different developmental stages of control/transgenic rice plants were ground in liquid $\mathrm{N}_{2}$ to fine powder, dissolved in the Trizol reagent, incubated at $15-30^{\circ} \mathrm{C}$ for $5 \mathrm{~min}$, mixed with chloroform $(0.2 \mathrm{~mL} / 1 \mathrm{~mL}$ Trizol reagent $)$, and centrifuged $12,000 \times g$ at $2-8{ }^{\circ} \mathrm{C}$ for $15 \mathrm{~min}$. The resulting supernatants were mixed with isopropanol $(0.5 \mathrm{~mL} / 1 \mathrm{~mL}$ Trizol reagent), incubated at $15-30^{\circ} \mathrm{C}$ for $10 \mathrm{~min}$, and centrifuged at $12,000 \times g$ for $10 \mathrm{~min}$ at $2-8^{\circ} \mathrm{C}$ to collect RNA pellets. After twice washing with $75 \%$ ethanol, the resulting RNA pellets were dried and resuspended in water or an appropriate buffer.

\section{Reverse transcriptase-PCR analysis}

First strand cDNAs were synthesized at $42^{\circ} \mathrm{C}$ for $1 \mathrm{~h}$ in a $20 \mu \mathrm{L}$ reaction that contains $2.0 \mu \mathrm{g}$ of total RNAs, $4.0 \mu \mathrm{L}$ of $5 \times$ reaction buffer, $1.0 \mu \mathrm{L}$ of oligo $\mathrm{d}(\mathrm{T}){ }_{15}(50 \mathrm{mmol} / \mathrm{L}), 2.0 \mu \mathrm{L}$ dNTP mix (10 mM each), $1.0 \mu \mathrm{L}$ Ribonuclease Inhibitor (40 U, TAKARA, Japan), $1 \mu \mathrm{L}$ AMV reverse transcriptase (5 U, TAKARA, Japan). $0.5 \mu \mathrm{L}$ of the reaction product was used for subsequent PCR amplification of gene-specific cDNA fragments in a $50 \mathrm{~mL}$ reaction containing $40 \mu \mathrm{L}$ of RNase-free $\mathrm{H}_{2} \mathrm{O}, 5 \mu \mathrm{L}$ of $10 \times$ PCR buffer, $1 \mu \mathrm{L}$ dNTP mix (10 mM each), $1 \mu \mathrm{L}$ of forward primer $(10 \mu \mathrm{mol} / \mathrm{L}), 1 \mu \mathrm{L}$ of reverse primer $(10 \mu \mathrm{mol} / \mathrm{L})$, and $0.4 \mu \mathrm{L}$ of DNA polymerase $(2.5 \mathrm{U} / \mu \mathrm{L})$. The gene-specific primer pairs used for the RT-PCR reactions were: gaagaagaagatgtcttgctg and acagtagcagcatccatacg for $O s M T 2 b$; gtgcttgtgtgtcattctaccc and ggacatggtggttcagttgaaccc for OsSRT1; tgggtctccatatatgttcac and ggactcattccgcgacttac for OsPDK1; gccaggctctgggtcag and cgggtcgcgecccacg for OsPDK2.

Table 1. Sequences of primers.

\begin{tabular}{|c|c|c|c|}
\hline Names of primers & abbreviation & sequence ( $5^{\prime}$ to $\left.3^{\prime}\right)$ & Description \\
\hline OsMT2b promoter $\mathrm{f}$ & P-MT-F & aaaaaagcttgagatgctaatcaagtctctctg & Hind III \\
\hline OsMT2b promoter $r$ & P-MT-R & aaaagatatcagatgttgttgctgattgagctc & EcoR V \\
\hline OsSRT1 promoter $\mathrm{f}$ & P-SRT-F & aaaagaattcgtgcttgtgtgtcattctaccc & EcoR I \\
\hline OsSRT1 promoter $r$ & P-SRT-R & aaaaggtaccggacatggtggttcagttgaaccc & Kpn I \\
\hline OsPDK1 promoter $\mathrm{f}$ & P-PDK1-F & aaaagaattcgtagtgtcaggctgtcagcaac & EcoR I \\
\hline OsPDK1 promoter $r$ & P-PDK1-R & aaaatctagaccctaccgacaacagcaccac & Xba I \\
\hline OsPDK2 promoter $\mathrm{f}$ & P-PDK2-F & aaaagaattccgctgtactatgagtcgtacc & EcoR I \\
\hline OsPDK2 promoter $r$ & P-PDK2-R & aaaaggtaccatcatgtagcgcaggctcac & Kpn I \\
\hline Ubi promoter $\mathrm{f}$ & P-Ubi-F & aaaaggatccagtgcagcgtgacccggtc & BamH I \\
\hline Ubi promoter $r$ & P-Ubi-R & aaaacccgggcagaagtaacaccaaacaacagg & Sma I \\
\hline OsMT2b RNAi 1 & R-MT-1 & aaaagaattcgctgctccatccaacaagg & EcoR I \\
\hline OsMT2b RNAi 2 & R-MT-2 & aaaagatatcgaagcctggcacgcatgagg & EcoR V \\
\hline OsMT2b RNAi 3 & R-MT-3 & aaaaactagtgaagcctggcacgcatgagg & Spe I \\
\hline OsSRT1 RNAi 1 & R-SRT-1 & aaaagtcgacggctgttcgagctcttccattg & Sal I \\
\hline OsSRT1 RNAi 2 & R-SRT-2 & aaaaggatccataccatcaagccccacaaccag & BamH I \\
\hline OsSRT1 RNAi 3 & R-SRT-3 & aaaaaagcttcataccatcaagccccacaaccag & Hind III \\
\hline OsPDK1 sense $\mathrm{f}$ & S-PDK1-F. & aaaagtcgactgggtctccatatatgttcac & Sal I \\
\hline OsPDK1 sense $r$ & S-PDK1-R & aaaaaagcttggactcattccgcgacttac & Hind III \\
\hline OsPDK2 sense $f$ & S-PDK2-R & aaaagtcgacgccaggctctgggtcag & Sal I \\
\hline OsPDK1 sense $r$ & S-PDK2-R & aaaaaagcttcgggtcgcgccccacg & Hind III \\
\hline
\end{tabular}


Table 2. Promoter locations.

\begin{tabular}{lllll}
\hline Promoters & BAC clones & Locations in the BAC & Gene names & Locations in the rice genome \\
\hline OSMT2b & AC079356 & $90035 \sim 91973$ & Os05g0111300 & $-49 \sim-1987$ \\
OSSRT1 & AL663014 & $143857 \sim 141928$ & Os04g0271000 & $-49 \sim-1978$ \\
OSPDK1 & AC082644 & $112200 \sim 113877$ & Os03g0370000 & $-24 \sim-1701$ \\
OSPDK2 & AP003749 & $87838 \sim 85738$ & Os07g0637300 & $+94 \sim-2006$ \\
\hline
\end{tabular}

Note: "+" means upstream of ATG and "-" means downstream of ATG.

doi:10.1371/journal.pone.0017444.t002

\section{RNA blot analysis}

For RNA blot hybridization, equal amounts $(\sim 20-30 \mu \mathrm{g})$ of total RNAs were separated on $1.2 \%$ denaturing agarose gels containing $12.5 \%$ formaldehyde and transferred on to a Hybond$\mathrm{N}$ nylon membrane (Amersham Biosciences). The hybridization probes were amplified by gene-specific primers used for RT-PCR analysis and were labelled using an $\left[\alpha_{-}^{32} \mathrm{P}\right]$-dCTP random primelabelling system. Hybridization was performed at $42^{\circ} \mathrm{C}$ following a previously described procedure [40]. After hybridization, the membrane was washed twice with $2 \times \mathrm{SSC}$ containing $0.1 \%$ SDS $(\mathrm{w} / \mathrm{v})$ and twice with $0.1 \times \mathrm{SSC}$ containing $0.1 \% \mathrm{SDS}(\mathrm{w} / \mathrm{v})$ at $50^{\circ} \mathrm{C}$, and the hybridization signals were visualized by Molecular Imager PharosFX Plus System (Bio-Rad).

\section{Immunoblot Analysis}

Tissues were collected from the transgenic and wild type plants, and total proteins were extracted as described [41]. The protein extracts (100 $\mu \mathrm{g}$ per lane) were separated by SDS-polyacrylamide gel electrophoresis and transferred to Pure Nitrocellulose Blotting Membrane (Pall Corporation) using the wet transfer apparatus. The membranes were incubated in blocking buffer $(5 \%[\mathrm{w} / \mathrm{v}]$ skimmed milk powder, 0.05\% [v/v] Tween 20, $20 \mathrm{mM}$ Tris-HCl, and $500 \mathrm{mM} \mathrm{NaCl}, \mathrm{pH} 7.5$ ) for $1 \mathrm{~h}$, washed 5 times ( 5 min each) with TBST $(0.05 \%[\mathrm{v} / \mathrm{v}]$ Tween $20,20 \mathrm{mM}$ Tris-HCl, and

\section{References}

1. The Arabidopsis Genome Initiative (2000) Analysis of the genome sequence of the flowering plant Arabidopsis thaliana. Nature 408: 796-815

2. Frantz J, Pinnock D, Klassen S, Bugbee B (2004) Rice: Characterizing the environmental response of a gibberellic acid-deficient rice for use as a model crop. Agron J 96: 1172-1181.

3. Rice Genome Annotation [http://rice.plantbiology.msu.edu/riceInfo/info. shtml\#Genes].

4. International Rice Genome Sequencing Project (2005) The map-based sequence of the rice genome. Nature 436: 793-800.

5. Pastuglia M, Azimzadeh J, Camilleri C, Bouchez D (2003) Forward and reverse genetics in Arabidopsis: isolation of cytoskeletal mutants. Cell Biol Intl 27: 249-250.

6. Bouchez D, Höfte H (1998) Functional genomics in plants. Plant Physiol 118: 725-732.

7. Upadhyaya NM (2007) Rice Functional Genomics - Challenges, Progress and Prospects. New York: Springer. 500 p.

8. An G (2008) T-DNA Tagging Lines. In: Hirono H, Sano Y, Hirai A, Sasaki T. Biotechnology in Agriculture and Forestry: Rice Biology in the Genomics Era. Vol. 62. New York: Springer. pp 95-106.

9. Bernstein E, Caudy A, Hammond S, Hannon G (2001) Role for a bidentate ribonuclease in the initiation step of RNA interference. Nature 409: 363-366.

10. Odell JT, Nagy F, Chua N-H (1985) Identification of DNA sequences required for activity of the cauliflower mosaic virus $35 \mathrm{~S}$ promoter. Nature 313: 810-812.

11. Christensen A, Sharrock R, Quail P (1992) Maize polyubiquitin genes: structure, thermal perturbation of expression and transcript splicing, and promoter activity following transfer to protoplasts by electroporation. Plant Mol Biol 18: 675-689.

12. Suwannaketchanatit C, Chaisuk P, Piluek J, Peyachoknakul S (2006) Evaluation of constitutive promoters for gene expression in Dendrobium protocorms and flowers. Kasetsart J (Nat Sci) 40: 934-943.

13. Rooke L, Byrne D, Salgueiro S (2000) Marker gene expression driven by the maize ubiquitin promoter in transgenic wheat. Ann Appl Biol 136: 167-172.
$500 \mathrm{mM} \mathrm{NaCl}, \mathrm{pH} \mathrm{7.5),} \mathrm{and} \mathrm{incubated} \mathrm{with} \mathrm{the} \mathrm{primary}$ antiserum (1:500 dilution) for $2 \mathrm{~h}$ at room temperature. After 5 rinses $(5 \mathrm{~min}$ each) with TBST, the membranes were incubated with the secondary antibody (alkaline phosphatase-conjugated goat anti-rabbit IgG [ALP], 1:10000 dilution; Kirkegaard and Perry Laboratories) for $1.5 \mathrm{~h}$ at room temperature, washed 5 times (5 min each) with TBST, and subsequently incubated in the substrate buffer $(0.33 \mathrm{mg} / \mathrm{mL}$ nitroblue tetrazolium [SigmaAldrich], $0.165 \mathrm{mg} / \mathrm{mL}$ BCIP [Bio-Basic], $0.1 \mathrm{M}$ Tris, $0.1 \mathrm{M}$ $\mathrm{NaCl}$, and $5 \mathrm{mM} \mathrm{MgCl}_{2}, \mathrm{pH} 9.5$ ) for several minutes in the dark, and the chemiluminescent signals were subsequently detected by autoradiography film.

\section{Supporting Information}

Text S1 Construction of RNAi/antisense transgenes. (DOC)

\section{Author Contributions}

Conceived and designed the experiments: CZ. Performed the experiments: Jing Li DJ HZ FL JY. Analyzed the data: DJ JY LH. Contributed reagents/materials/analysis tools: Z. Li HZ XF. Wrote the paper: Jing Li DJ CZ Jianming Li Z. Liu.

14. Nakatsuka T, Pitaksutheepong C, Yamamura S, Nishihara M (2007) Induction of differential flower pigmentation patterns by RNAi using promoters with distinct tissue-specific activity. Plant Biotechnol Rep 1: 251-257.

15. Hirsche J, Engelke T, Völler D, Götz M, Roitsch T (2009) Interspecies compatibility of the anther specific cell wall invertase promoters from Arabidopsis and tobacco for generating male sterile plants. Theor Appl Genet 118: 235-345.

16. Masclaux FG, Charpenteau M, Takahashi T, Pont-Lezica R, Galaud J-P (2004) Gene silencing using a heat-inducible RNAi system in Arabidopsis. Biochem Biophys Res Commun 321: 364-369.

17. Gatz C, Frohberg C, Wendenburg R (1992) Stringent repression and homogeneous derepression by tetracycline of a modified CaMV $35 \mathrm{~S}$ promoter in intact transgenic tobacco plants. Plant J 2: 397-404.

18. Mett VL, Lochhead LP, Reynolds PH (1993) Copper-controllable gene expression system for whole plants. Proc Natl Acad Sci U S A 90: 4567-4571.

19. Ait-Ali T, Rands C, Harberd NP (2003) Flexible control of plant architecture and yield via switchable expression of Arabidopsis gai. Plant Biotechnol J 1: 337-343.

20. Zuo J, Chua NH (2000) Chemical-inducible systems for regulated expression of plant genes. Curr Opin Biotechnol 11: 146-151.

21. Vasil IK (1999) Molecular Improvement of Cereal Crops. Dordrecht: Kluwer Academic Publishers. 500 p.

22. Yuan J, Chen D, Ren Y, Zhang X, Zhao J (2007) Characteristic and expression analysis of a metallothionein gene, OsMT2b, down-regulated by cytokinin suggests functions in root development and seed embryo germination of rice. Plant Physiol 146: 1637-1650.

23. Huang L, Sun Q, Qin F, Li C, Zhao Y, et al. (2007) Down-regulation of a SILENT INFORMATION REGULATOR2-related histone deacetylase gene, OsSRT1, induces DNA fragmentation and cell death in rice. Plant Physiol 144: 1508-1519.

24. Jan A, Nakamura H, Handa H, Ichikawa H (2006) Gibberellin regulates mitochondrial pyruvate dehydrogenase activity in rice. Plant Cell Physiol 47: $244-253$. 
25. Cherian MG, Jayasuryab A, Bay B-H (2003) Metallothioneins in human tumors and potential roles in carcinogenesis. Mutat Res/Fundam Mol Mech Mutage 533: 201-209.

26. Leszczyszyn OI, Schmid R, Blindauer CA (2007) Toward a property/function relationship for metallothioneins: Histidine coordination and unusual cluster composition in a zinc-metallothionein from plants. Proteins 68: 922-935.

27. Wong HL, Sakamoto T, Kawasaki T, Umemura K, Shimamoto K (2004) Down-regulation of metallothionein, a reactive oxygen scavenger, by the small GTPase OsRac1 in rice. Plant Physiol 135: 1447-1456.

28. Steffens B, Sauter M (2009) Epidermal cell death in rice is confined to cells with a distinct molecular identity and is mediated by ethylene and $\mathrm{H}_{2} \mathrm{O}_{2}$ through an autoamplified signal pathway. Plant Cell 21: 184-196.

29. Smith JS, Brachmann CB, Celic I, Kenna MA, Muhammadi S, et al. (2000) A phylogenetically conserved $\mathrm{NAD}^{+}$-dependent protein deacetylase activity in the Sir2 protein family. Proc Natl Acad Sci U S A 97: 6658-6663.

30. Brachmann CB, Sherman JM, Devine SE, Cameron EE, Pillus L, et al. (1995) The SIR2 gene family, conserved from bacteria to humans, functions in silencing, cell cycle progression, and chromosome stability. Genes Dev 9: 2888-2902.

31. Fu W, Wu K, Duan J (2007) Sequence and expression analysis of histone deacetylases in rice. Biochem Biophys Res Commun 356: 843-850.

32. Good L (2003) Translation repression by antisense sequences. Cell Mol Life Sci 60: $854-861$.

33. Praveena S, Mishra AK, Dasgupta A (2005) Antisense suppression of replicase gene expression recovers tomato plants from leaf curl virus infection. Plant Sci 168: 1011-1014.
34. Coopera SR, Taylora JK, Miragliaa LJ, Dean NM (1999) Pharmacology of antisense oligonucleotide inhibitors of orotein expression. Pharmacol Ther 82: 427-435.

35. van der Krol AR, Mur LA, de Lange P, Mol JNM, Stuitje AR (1990) Inhibition of flower pigmentation by antisense CHS genes: promoter and minimal sequence requirements for the antisense effect. Plant Mol Biol 14: 457-466.

36. Lin H, Ouyang S, Egan A, Nobuta K, Haas BJ, et al. (2008) Characterization of paralogous protein families in rice. BMC Plant Biol 8: 18.

37. Elomaa P, Helariutta Y, Kotilainen M, Teeri TH (1996) Transformation of antisense constructs of the chalcone synthase gene superfamily into Gerbera hybrida: differential effect on the expression of family members. Molecular Breed 2: $41-50$.

38. Wu L, Zhang Q, Zhou H, Ni F, Wu X, et al. (2009) Rice microRNA effector complexes and targets. Plant Cell 21: 3421-3435

39. Hiei Y, Ohta S, Komari T, Kumashiro T (1994) Efficient transformation of rice (Oryza sativa L.) mediated by Agrobacterium and sequence analysis of the boundaries of the T-DNA. Plant J 6: 271-282.

40. Yadetie F, Sandvik AK, Bergum H, Norsett K, Laegreidl A (2004) Miniaturized fluorescent RNA dot blot method for rapid quantitation of gene expression. BMC Biotechnol 4: 12.

41. Yu S-M, Kuo Y-H, Sheu G, Sheu Y-J, Liu L-F (1991) Metabolic derepression of alpha-amylase gene expression in suspension-cultured cells of rice. J Biol Chem 266: 21131-21137. 